\title{
A Case Study of Disseminated Histoplasmosis Linked to Common Variable Immunodeficiency
}

Acir Rachid, Lucila Stange Rezende

Suzelle Freitas de Moura, Paulo C. Loffy

and Francisco Luiz Gomide M. Magalhães

\author{
Federal University at Paraná, Curitiba, PR, Brazil
}

\begin{abstract}
Histoplasma capsulatum, a ground fungus, can infect humans, normally in endemic areas; the resulting disease can be asymptomatic or it can have a benign development, but in rare cases it can develop into a serious clinical condition and can even be fatal. Its most characteristic initial location is in the lungs, resembling tuberculosis, often accompanied by mediastinitis and an exuberant fibrotic response. The spread of this infection can be caused by the concomitance of another illness that alters the immunological balance. Sometimes such an association is not clear. Therefore, disseminated histoplasmosis is defined as a clinical condition where the fungus is present in more than one location. Common variable immunodeficiency is characterized by a generalized failure in the synthesis of antibodies, leading the affected individuals to present recurrent infections, especially those caused by encapsulated bacteria, most often involving the respiratory tract. We studied a serious case of disseminated histoplasmosis, accompanied by common variable immunodeficiency, observed at the Infirmary of the department of Medical Practice of the Federal University at Paraná Hospital das Clínicas.

Key Words: Disseminated histoplasmosis, common variable immunodeficiency, immunoglobulins.
\end{abstract}

\section{Clinical Case}

The patient, E.M.P., 29 years of age, male, widowed, a native of Curitiba, Paraná, arriving from Rio de Janeiro, was admitted at the Federal University at Paraná Hospital das Clínicas with symptoms of weight loss and chronic diarrhea. The patient had started a progressive condition, 60 days earlier, of weight loss (approximately $30 \mathrm{~kg}$ ), a dry cough, night sweats, and diarrhea (which began 40 days earlier). The patient concurrently presented right bronchial paresis, aphasia, and lesions in the oropharyngeal cavities suggestive of moniliasis. The patient's partner had died from complications related to HIV infection. The patient

Received on 18/06/2002; revised 10/01/2002.

Address for correspondence: Dr. Acir Rachid. Alameda Princesa Isabel, 1223, 7o. andar, ap. 71, Curitiba, Paraná, Brazil. Zip code: 80730-080. Phone: (55 41) 222-0357.

The Brazilian Journal of Infectious Diseases 2003;7(4):268-272 (C) 2003 by The Brazilian Journal of Infectious Diseases and Contexto Publishing. All rights reserved. denied using tobacco and/or illicit drugs. A physical examination revealed a fair general state of health; The patient was cachectic, discolored, dehydrated, and aphasic. Vital signs were stable. The patient had whitish, necrotic lesions on the tongue, as well as cervical lymph node enlargement. There was reduced pulmonary elasticity, and a diffusely diminished vesicular murmur, without adventitious noise. The patient had right bronchial monoparesis (grade III muscle power) and aphasia of expression. Three anti-HIV results with ELISA and P24 antigen search were negative; serology for Paracoccidioidomycosis was negative; VDRL was not reactive; leukocyte count, 6400 cells / $\mathrm{ml} ; \mathrm{CD}_{4} / \mathrm{CD}_{8}$ : 0.23; VHS was $65 \mathrm{~mm}$ in $1 \mathrm{hr}$. Cerebrospinal fluid showed hyperproteinorachia. A chest X-ray showed a perihilar lesion on the left, and a chest CT scan showed nodular lesions of various sizes; the one located in the anterior segment of the superior lobe was the central nodular lesion, with hilar involvement. An abdominal sonogram showed multiple augmented retroperitoneal lymph nodes and a thickening of the cecum wall. Colonoscopy presented a vegetating ulcerous lesion, 
friable at the touch of the instrument, which deformed the ileocecal valve; a retrograde exam showed various shallow ulcerations, with irregular edges and a fibrinous background on the ascendant colon; the hepatic angle was transversal; the colon was descendent and sigmoid. Serial biopsies were made, which showed chronic and acute ulcerated colitis, secondary to deep mycosis, suggestive of histoplasmosis (attached). Anatomic/pathologic exam of cervical lymph nodes showed a proliferative, atypical process, and epithelioid granulomatous chronic lymphnoditis - a histological condition highly suggestive of deep mycosis (attached). A culture for fungus of the biopsy material showed the development of Histoplasma capsulatum. A cranium CT Scan showed a hypodense lesion on the left parietal lobe, suggestive of a sequel (prior history of cranialencephalic trauma) and volumetric reduction of the cerebral hemispheres. Encephalic MRI showed right maxillary sinusopathy, volumetric reduction of the encephalic parenchyma, and areas with anomalous spots in the frontal-parietal regions, which may represent areas of necrosis and/or ischemia, with dilatation of the left, lateral ventricle. The levels of $\mathrm{IgG}$ $<17.2 \mathrm{mg} / \mathrm{dl}, \operatorname{IgA}=93.1 \mathrm{mg} / \mathrm{dl}$, and $\operatorname{IgM}=401 \mathrm{mg} / \mathrm{dl}$ were compatible with a hypothesis of common variable immunodeficiency.Methicillin-resistant Staphylococcus aureus was detected in a culture of nasal secretion, and Acinetobacter baumanii, sensitive to imipenem, was found in the urine culture. Treatment with Amphotericin B was begun, but the patient's state deteriorated progressively, leading to death.

\section{Discussion}

Histoplasma capsulatum is a dimorphic fungus that exists in nature in the mycelium phase and turns into yeast at human body temperature. It is distributed worldwide, but this fungus is more prevalent in certain areas of North and South America. It was originally a problem of rural areas, but today it has emerged in many urban areas, probably due to an increase in the number of pigeons and bats, animals in whose feces this fungus can be found [1,2].
Contamination occurs through inhalation of spores or hyphae and, depending on the immunological condition of the individual, acute pulmonary histoplasmosis may arise, resulting in fever, hypoxia, and pulmonary infiltrates. The chronic form of pulmonary histoplasmosis may appear if the individual already has a pre-existing pulmonary disease, such as emphysema, or the disseminated form of histoplasmosis may develop, which occurs in people with immunodeficiencies, especially HIV; this disseminated form is rapidly fatal and cannot be treated [3,4]. Usually only $5 \%$ of individuals develop the illness after a light exposure to the fungus [5].

Acute pulmonary histoplasmosis may develop in normal individuals after heavy exposure to the fungus. In almost $80 \%$ of cases the infection is self-limiting. Clinical manifestations are fever, tremors, cephalalgia, myalgia, anorexia, cough, and chest pain. Many people improve in a few weeks, but some may suffer fatigue for several months [6].

Individuals suffering from the chronic form of histoplasmosis are usually affected by a pre-existing pulmonary disease [7]. They present productive cough, dyspnea, chest pain, fatigue, fever, and they show fibrotic infiltrates with cavitations in chest X-rays or CT scans. Chronic pulmonary histoplasmosis is progressive, and manifests itself through an increase in the cavitations, the formation of new cavities, and the formation of bronchial-pleural fistulae [8].

Although hematogenic dissemination of the fungus occurs in most patients during acute illness, disseminated histoplasmosis only occurs in individuals with immunodeficiency at an early or at an advanced age [9]. In children or in people with severe immunodeficiencies, an acute and rapidly fatal evolution with diffuse reticular-endothelial involvement is observed. A more chronic evolution is observed in adults that are not severely immunodeficient.

Disseminated histoplasmosis is classically described in immunodeficient patients, especially those with AIDS, patients that are at an either early or an advanced age [10-12], and those suffering from idiopathic $\mathrm{CD}_{4}$ lymphocytopenia. In approximately $1 / 3$ to $2 / 3$ of the cases no identifiable risk factors are recognized in the dissemination of the illness [13]. 
The disseminated form of this deep mycosis has various clinical manifestations (Table 1).

Table 1. Clinical findings of disseminated histoplasmosis. Frequency of the main clinical findings in 66 cases of disseminated histoplasmosis. (Data from Sathapayongs et al. 1983) [3].

\begin{tabular}{lc}
\hline Manifestations & Percentage \\
\hline Fever & 100 \\
Pulmonary illness & 70 \\
Weight loss & 60 \\
Hepatomegaly & 60 \\
Neurological symptoms & 40 \\
Splenomegaly & 40 \\
Lymphadenopathy & 30 \\
Meningitis & 5 \\
\hline
\end{tabular}

Other manifestations include gastrointestinal involvement, such as ulcerations or polypoid masses involving the colon and/or ileum, especially the ileocecal valve [14].

Common variable immunodeficiency (CVI) is a rare pathology, with an incidence of approximately 1 in every 100,000 individuals, and it affects people in equal proportions among the sexes [15]. It is the most common immunodeficiency syndrome among adults, and it affects individuals of any age; but in most cases this illness is not clinically apparent before the second or third decade of life $[16,17]$. Common variable immunodeficiency has been described as an idiopathic illness that manifests itself during life for some reason, but there is a general agreement among specialists that the defect exists from birth. Rarely, CVI may develop after fetal Rubella or postnatal infection by the EpsteinBar virus [18]. A period of several years between the beginning of infection and the establishment of a CVI diagnosis is typical [19].

There are records of several related individuals affected by CVI or selective IgA deficiency, which has led to the suggestion that this humoral immunodeficiency is genetically determined [7]. Recent studies show a high prevalence of two extended haplotypes of the MHC-III (Major Histocompatibility Complex class III) containing rare deletions of the $\mathrm{C} 2 \mathrm{and} / \mathrm{or} \mathrm{C} 4 \mathrm{a}$ alleles in groups of relatives, at least one individual of which was affected by CVI or by IgA deficiency, and a high prevalence of these haplotypes in affected individuals [20,21]. These studies suggest that a susceptible gene (or genes) for both these immunodeficiencies is located within the MHC class III region in chromosome 6 , or near it.

Other studies associate CVI with the occurrence of an alanine or a neutral valine in position 57 of the DQ beta chain. This residue could impair the appearance of antigens or the capacity for joint interaction between $B$ and T cells, which would result in a deficiency of the immune system [22]. The mechanism through which a gene or genes in the MHC class III or II region become susceptible to CVI is still unknown.

The distinguishing mark of CVI is a significant decrease in the IgG serum concentration, which is invariably associated with a significant decrease in the IgM and IgA serum concentrations. Most patients with CVI have a number of BV cells, surrounding and in normal peripheral lymphoid tissues. Several immunological abnormalities have been identified in these patients through in vitro tests.

Studies have demonstrated that patients with CVI have impaired secretion of one or more immunoglobulin isotypes, with the secretion of $\operatorname{IgF}$ and $\operatorname{IgA}$ usually being more affected than IgM [23,24]. They also showed that the B cells of certain patients with CVI may not be intrinsically abnormal and may only need appropriate stimulation (which is presumably absent in vivo) for them to secrete immunoglobulins appropriately.

Many patients have abnormalities in the T cells, when examined in vitro. About half of the patients have selective abnormalities of T cell activation. In these cells there is a decrease in the activity of the genes directing the synthesis of IL-2, IL-4, and IL-5 (the high serum level of these interleukins in patients with CVI is associated with chronic immune activation and a low number of $\mathrm{CD}_{40}$ lymphocytes) [25]. Both the $\mathrm{CD}_{40}$ ligand and the interleukins are essential for $T$ cells to help B cells, which leads to the idea that in some cases the fundamental defect in CVI is in the T cells [26-28]. 
The malfunction of the immune response observed in idiopathic $\mathrm{CD}_{4}$ lymphocytopenia (a rare condition in which $\mathrm{CD}_{4}$ cells are at lower than normal levels and immunoglobulins areat the lower limit of normality, different from HIV infection, where there is a condition of hypogammaglobulinemia) is similar to the response found in CVI, which suggests that at least one subgroup of patients with CVI and idiopathic $\mathrm{CD}_{4}$ lymphocytopenia shares common pathogenetic paths [29].

Common variable immunodeficiency is not just a syndrome of ineffective immunoglobulin secretion, but rather it is a complex condition of disorder between $\mathrm{B}$ and T cells. The distinguishing clinical attribute of CVI is recurrent bacterial infections caused by encapsulated bacteria. Sinusitis, otitis media, bronchitis, and pneumonia are the most common infections observed. In untreated patients, chronic sinusitis and bronchiectasis are frequent complications [30].

A syndrome that is unique to enteroviral meningoencephalitis is observed in these patients with hypogammaglobulinemia. This syndrome is clinically characterized by a progressive neurological deterioration. The most common enteroviral pathogen is echovirus 11 . Usually this syndrome is progressive and fatal [31].

With CVI, there is a higher risk of developing an autoimmune illness, with an increase in incidence of approximately $20 \%$. The most common autoimmune illnesses are mediated hemolytic anemia from antibodies and thrombocytopenia, which can occur in approximately $10 \%$ of patients. Neutropenia may also occur. Patients with CVI suffer from a large variety of infectious and non-infectious gastrointestinal illnesses [16,17].

Approximately $60 \%$ of untreated patients develop diarrhea. The incidence of ulcerative colitis and Crohn's disease increases with CVI. Approximately $10 \%$ of patients will develop malabsorptionillnesses with weight loss that may be debilitating.

Patients with CVI have a higher incidence of lymphoproliferative lesions. More common than Lymphomais the occurrence of benignlymphoproliferative lesions. Approximately $1 / 3$ of patients will have Splenomegaly or diffuse lymphadenopathy, or both.
Common variable immunodeficiency therefore comprises a heterogeneous group of immunological disorders characterized by a generalized diminution of the synthesis of antibodies, which classically predisposes the individual to recurrent bacterial infections, especially involving the superior and inferior respiratory tracts.

Treatment with Amphotericin B did not lead to therapeutic success in our patient, as the patient died. It is known that the mortality of disseminated histoplasmosis in severely affected patients is close to $50 \%$ [17].

The CVI diagnosis is confirmed in patients with recurrent infections who have low $\operatorname{IgG}$, IgM, and IgA doses compared to the standard values for each age range. A diminished or absent antibody response to immunization concludes the diagnosis. Treatment consists of monthly replacement of EV gamma globulin and the early use of antibiotics at the first signs of infection $[32,33]$.

This case study concerns an atypical manifestation of systemic infection in a patient with Common Variable Immunodeficiency. Far from representing a conclusive elucidation of the issue, this study adds to the discussion of the pathogenesis of this syndrome by raising questions about which factors involved in the failure of cellular immunity predispose individuals to develop the disseminated form of this deep mycosis.

\section{References}

1. Eisenstein E.M., Sneller M.C. Common variable immunodeficiency: diagnosis and management. Annals of Allergy 1994;73:285-92.

2. Goodwin R.A. Jr., Shapiro J.L., Thurman G.H., et al. Disseminated histoplasmosis: Clinical and pathologic correlations. Medicine (Baltimore) 1980;59:1.

3. Sathapatayavings B., Batteiger B.E., Wheat L.J., et al. Clinical and laboratory features of disseminated histoplasmosis during two large urban outbreaks. Medicine (Baltimore) 1983;62:263.

4. Wheat L.J., Connolly-Stringfield P.A., Baker R.L., et al. Disseminated histoplasmosis in the acquired immune deficiency syndrome: Clinical findings, diagnosis and treatment, and review of the literature. Medicine (Baltimore) 1990;69:361.

5. Wheat L.J., Slama T.G., Norton J.A., et al. Risk factors for disseminated or fatal histoplasmosis. Ann Intern Med 1982;96:159. 
6. Wheat J. UpToDate.

7. Sander C.A., Medeiros L.J., Weiss L.M., et al. Lymphoproliferative lesions in patients with common variable immunodeficiency syndrome. Am J Surg Pathol 1992; $16: 1170-82$.

8. Wheat L.J., Batteiger B.E., Sathapatayavongs B. Histoplasma capsulatum infections of the central nervous system: A clinical review. Medicine (Baltimore) 1990;69:244.

9. Asherton G..L. Late onset hypogammaglobulinemia, In: Asherton G..L., Webster A.D.B., eds. Diagnosis and treatment of immunodeficiency diseases. Blackwell Scientific Publications 1980:37-60.

10. Hermans P., Diax-Bruxo J., Stobo J. Idiopathic late-onset immunoglobulin deficiency. Am J Med 1976;61:221-33.

11. Cunningham-Rundles C. Clinical and immunological analyses of 103 patients with common variable immunodeficiency. J Clin Immunol 1989;9:22-33.

12. Smith D.K., Neal J.J., Holmberg S. Unexplained opportunistic infections and CD4 T-lymphocytopenia without HIV infection. The New England Journal of Medicine 1993;328:373-80.

13. Famularo G., Giacomelli R., Simone C., Tonietti G. The syndrome of idiopathic CD4+ lymphocytopenia. Ann Ital Med Int 1994;9:22-6.

14. Malone J.L., Simms T.E., Gray G.C., et al. Sources of variability in repeated T-helper lymphocyte counts from human immunodeficiency virus type 1-infected patients: total lymphocyte count fluctuations and diurnal cycle are important. J Acquir Immune Defic Syndr 1990;3:144-51.

15. Malone J.L., Oldfield E.C., Wagner K.F., et al. Abnormalities of morning serum cortisol and circadian rhythms of CD4+ lymphocyte counts in human immunodeficiency type 1 -infected adult patients. J Infect Dis 1992; 165:185-6.

16. Reichrt T., De Bruyere M., Deneys V., et al. Lymphocyte subset reference ranges in adult Caucasians. Clin Immunol Immunopathol 1991;60:190-208.

17. Wheat L. Histoplasmosis in the acquired immunodeficiency syndrome. Curr Top Med Mycol 1996;7:7. 